\title{
Shape Similarity Measurement for Boundary Based Features
}

\author{
Nafiz Arica ${ }^{1}$ and Fatos T. Yarman Vural ${ }^{2}$ \\ ${ }^{1}$ Department of Computer Engineering, Turkish Naval Academy \\ 34942, Tuzla, Istanbul, Turkey \\ narica@dho.edu.tr \\ ${ }^{2}$ Department of Computer Engineering, Middle East Technical University, \\ 06531 Ankara, Turkey \\ vuraleceng.metu.edu.tr
}

\begin{abstract}
In this study, we propose two algorithms for measuring the distance between shape boundaries. In the algorithms, shape boundary is represented by the Beam Angle Statistics (BAS), which maps 2-D shape information into a set of 1-D functions. Firstly, we adopt Dynamic Time Warping method to develop an efficient distance calculation scheme, which is consistent with the human visual system in perceiving shape similarity. Since the starting point of the representations may differ in shapes, the best correspondence of items is found by shifting one of the feature vectors. Secondly, we propose an approximate solution, which utilizes the cyclic nature of the shape boundary and eliminates the shifting operation. The proposed method measures the distance between the features approximately and decreases the time complexity substantially. The experiments performed on MPEG-7 Shape database show that both algorithms using BAS features outperform all the available methods in the literature.
\end{abstract}

\section{Introduction}

In Content Based Image Retrieval Systems (CBIR), the major goal is to search all the images in the database that are "similar" to a query image according to some predefined criterion. Usually, the images within a given distance from the query or the first few images that have the smallest distance to the query are retrieved as a result of the query operation. The similarity is measured by a distance function defined over the feature space of the images. The objective in most of the similarity measurement methods is to minimize the distance between two vectors by allowing deformations.

Although a sound mathematical basis exists, similarity measurement between the shape features is a serious problem in the shape retrieval and indexing applications. The shapes, which are visually similar, may not be "close" to each other in the vector space, defined over a particular distance measure. Another problem in similarity measurement is the complexity of the algorithms. Since the distance is calculated between the query and each database shapes, the time complexity of the process is crucial for large databases. Current studies on boundary based shape similarity use exhaustive search in matching the starting point of the shape descriptors so that the 
minimum distance can be found [1], [4], [5]. The problem gets even more complicated, when the dimension of the vector space is large.

The first motivation in this paper is to develop an efficient distance calculation method, which is consistent with the human visual system in perceiving similarity and possesses a remarkable robustness to distortions. For this purpose, we adopt Dynamic Time Warping algorithm, to the boundary based shape features. The shape boundary is represented using the Beam Angle Statistics (BAS) method, which represents 2-D shape information with a set of 1-D functions.

It is well-known that the starting point of the representations may differ in shapes. The proposed algorithm finds the best correspondence of items by shifting one of the feature vectors. However, this process increases the time complexity of the algorithm so that it may not be practical to search for the optimal solution especially for the database applications, which require large number of comparisons. For this reason, instead of finding the exact distance, the suboptimal solutions which measures the distance between the features approximately, may be more appropriate. Secondly, we develop an efficient method which utilizes the cyclic nature of the shape boundary and eliminates the shifting operation.

The performances of algorithms are tested using the data set of MPEG 7 Core Experiments Shape-1 Part B, which is the main part of the Core Experiments. The experiments show that the proposed similarity measurement algorithm outperforms the available methods in the literature.

The paper is organized as follows. Section 2 provides a summary of the shape representation based on BAS, proposed in [1]. Section 3 describes an improved version of Dynamic Time Warping algorithm used for the similarity measurement of BAS features. Section 4 explains a fast cyclic measurement between shape boundaries. The experiments performed on MPEG-7 Shape Database are presented in section 5. Finally, the last section concludes the paper and proposes future studies.

\section{Shape Representation Based on Beam Angle Statistics (BAS)}

BAS is based on the beams, which are the lines connecting the reference point with the rest of the points on the boundary [1]. The characteristics of each boundary point are extracted by using the beam angles in a set of neighborhood systems. The angle between each pair of beams is taken as the random variable at each point on the boundary. Then, the moments provide the statistical information about the shape. In the first moment, each valley and hill corresponds to a concave and convex visual part of the object shape. The other moments increase the discriminative power of the representation. An example of the proposed representation is shown in figure 1.

The BAS representation eliminates the use of any heuristic rule or empirical threshold value of shape boundaries in a predefined scale. BAS, also, gives globally discriminative features to each boundary point by using all other boundary points. Another advantage of BAS representation is its simplicity, yet consistency with the human perception through preserving visual parts of the shapes. The representation is scale, rotation and translation invariant. It is also insensitive to noise and occlusion. The details of the BAS representation can be found in [1]. 
Next step in shape description is to describe the 1-D BAS functions in a compact way. For this purpose, we adopt piecewise constant approximation method. The BAS function is segmented into equal size frames and each frame is represented by the segment average. Intuitively, in order to reduce the dimension of BAS function from $\mathrm{N}$ to $\mathrm{T}$, the data is divided into $\mathrm{T}$ equal-size frames. The average value of the data falling within a frame is calculated and the value obtained at each segment becomes the entries of the feature vector with dimension $\mathrm{T}$.

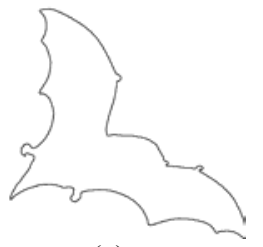

(a)

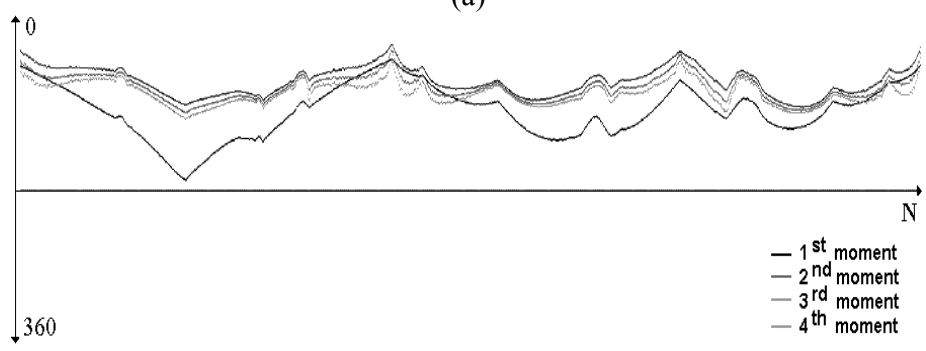

(b)

Fig. 1. (a) A sample shape boundary and (b) its fourth order statistics of Beam Angle.

\section{Dynamic Warping with Penalty}

The matching process needs to compensate the slight variations on the BAS functions appropriately by compressing the function at some places and expanding it at others. The classical DW algorithm achieves this goal by finding an optimal match between two sequences, which allows stretching and compression of the sequences.

In order to align two sequences, $\boldsymbol{A}=A_{1}, \ldots, A_{N}$ and $\boldsymbol{B}=B_{1}, \ldots, B_{M}$ using DW, we construct an $N$-by- $M$ matrix, where each element $(i, j)$ contains the distance between the points $A_{i}$ and $B_{j}$. The goal is to find a path through the matrix, which minimizes the sum of the local distances of the points, starting from $(1,1)$ and ending at $(N, M)$. This path is called warping path. If $D(i, j)$ is the global distance up to $(i, j)$ and the local distance at $(i, j)$ is given by $d(i, j)$, then DW algorithm uses the following recurrence relation:

$$
D(i, j)=d\left(A_{i}, B_{j}\right)+\min \left\{\begin{array}{c}
D(i-1, j-1), \\
D(i-1, j), \\
D(i, j-1) .
\end{array}\right.
$$


Given $D(1,1)=d\left(A_{1}, B_{l}\right)$ as the initial condition, we have the basis for an efficient recursive algorithm for computing $D(i, j)$. The algorithms starts from $D(1,1)$ and iterates through the matrix by summing the partial distances until $D(N, M)$, which is the overall matching score of the sequences $A$ and $B$. DW is accomplished with a time and space complexity of $O(N M)$.

DW algorithm as described above, while not permitting changes in the ordering of the sequence items, allows unconstrained compression and expansion of the items of the two sequences. This may be suitable for some applications, such as speech recognition, where the sequences of the same class may be traced out more slowly during one portion of the speech and more quickly during another portion. In order to allow such variations, it is necessary to distort the time axis appropriately. For this reason, the DW algorithms used for speech recognition does not need to penalize the expansion and compression of the sequences in the matching process. However, in shape boundary, the free expansion and compression of some part of the sequence change the visual appearance of the shape. These variations may carry meaningful information used to distinguish visually different parts of the shape boundary.

The above discussion leads us to the consideration of constraining the warping path to limit the amount of compression and expansion to a certain extent in the matching process. In this study, we propose to assign penalties for expansion and compression of the BAS function. For this purpose, the horizontal and vertical moves in the DW matrix are penalized by a constant. This modification improves the performance of the similarity measurements in BAS descriptors. The proposed Dynamic Warping with Penalty (DWP) algorithm for calculation of distance between two BAS vectors $\Gamma_{\mathrm{q}}$ and $\Gamma_{\mathrm{t}}$ is given below:

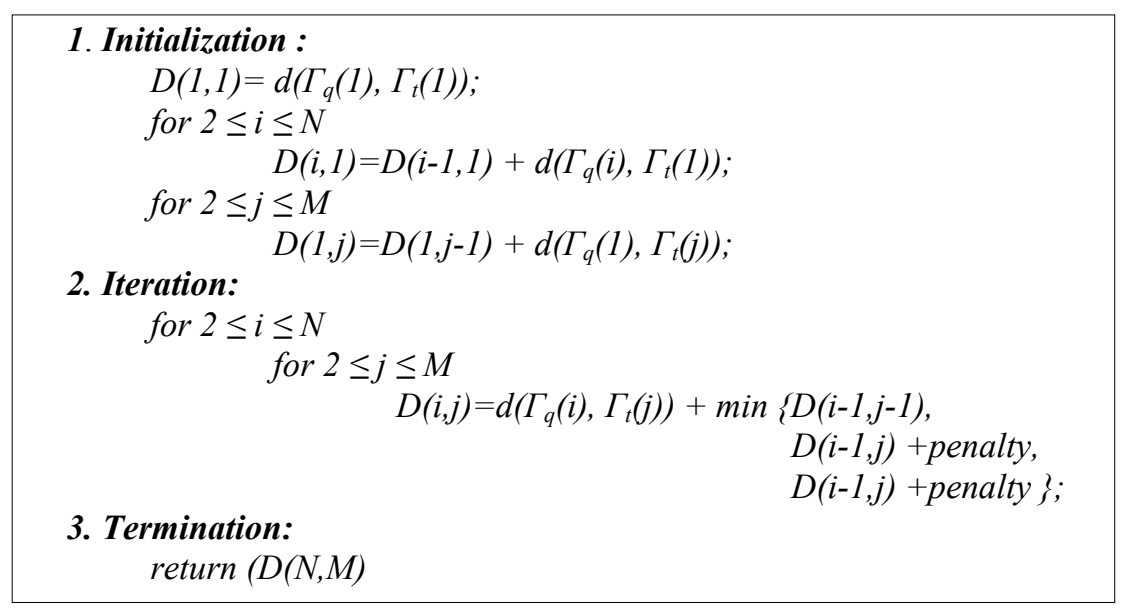

\section{Cyclic Sequence Comparison}

The BAS function, which is based on the shape boundary, is considered as a cyclic sequence. In order to align two BAS feature vectors, the starting boundary point is to 
be matched. This requires to define a unique starting point for each shape, which is not practically possible. For this reason, the alignment computation must determine the amount of cyclic shift that has taken place in order to find the optimal match.

The easiest method of solving cyclic sequence comparison problem is to shift any of the sequences one item at a time and recompute the alignment. The optimal alignment is, then, found by the cyclic shift which results with a minimum distance [1], [2]. However, shifting the elements of any sequence at each time, makes the complexity of the algorithm $O\left(M N^{2}\right)$ for the sequences with lengths $M$ and $N$.

Searching for strict optimality is not practical and efficient in image databases, which contain large number of shapes. Therefore, in practical problems, it is worth to find a suboptimal solution by approximate distance measures, rather than exact solution. The approximate approaches [6], [7], [8], double one of the sequences and then find the subsequence therein that best resembles the other sequence, which computes in time $O(M N)$.

In this study, we, also, approximate the optimal solution for the sack of efficiency and improve the method proposed in [8]. The original algorithm is developed for the partial matching of shape boundaries. However, our aim in BAS comparison is to find a matching between all the items of feature vectors. Thus, we improve the performance of the algorithm by assigning penalties in order to control the length of the warping path in the algorithm.

Given two cyclic sequences $\boldsymbol{A}$ and $\boldsymbol{B}$ with lengths $\mathrm{N}$ and M respectively, a minimum distance table with $\mathrm{M}$ columns and $2 \mathrm{~N}$ rows constructed by concatenating the sequence $\mathrm{A}$ with itself $\left(\mathrm{A}^{2}\right)$. Paths start from the first $\mathrm{N}$ entries of the first column and end at various points in the last column. $D(i, j)$ is defined as the total distance on the minimum distance path from the $(i, j)$ entry of the distance table to the end of the path at some point in the last column. The value of $D(i, j)$ is evaluated as;

$$
D(i, j)=\min \left\{\begin{array}{c}
d\left(A_{i+1}, B_{j+1}\right)+D(i+1, j+1), \\
d\left(A_{i+1}, B_{j}\right)+D(i+1, j)+\text { penalty, } \\
d\left(A_{i}, B_{j+1}\right)+D(i, j+1)+\text { penalty, }
\end{array}\right.
$$

for $i=1, \ldots, 2 \mathrm{~N}-1$ and $j=1, \ldots \mathrm{M}-1$. The boundary conditions are

$$
\begin{gathered}
D(i, M)=0, \quad i=1, \ldots, 2 N \quad \text { and } \\
D(2 N, j)=d\left(A_{2 N}, B_{j+1}\right)+D(2 N, j+1), \quad j=1, \ldots, M-1 .
\end{gathered}
$$

Finally let

$$
D(i, 0)=d(i, 1)+D(i, 1) .
$$

The values of $D(i, 0), i=1, \ldots N$ are the total distances of the paths through the minimum distance table from each starting point $i$ in A running from the first point to the last point of B. The path with the lowest $D(i, 0)$ is the minimum distance path in the table. 
Now, we are ready to give CSC algorithm for the BAS features. Given two BAS feature vectors $\Gamma_{\mathrm{q}}$ and $\Gamma_{\mathrm{t}}$, the cyclic sequence comparison method can be summarized by the following algorithm:

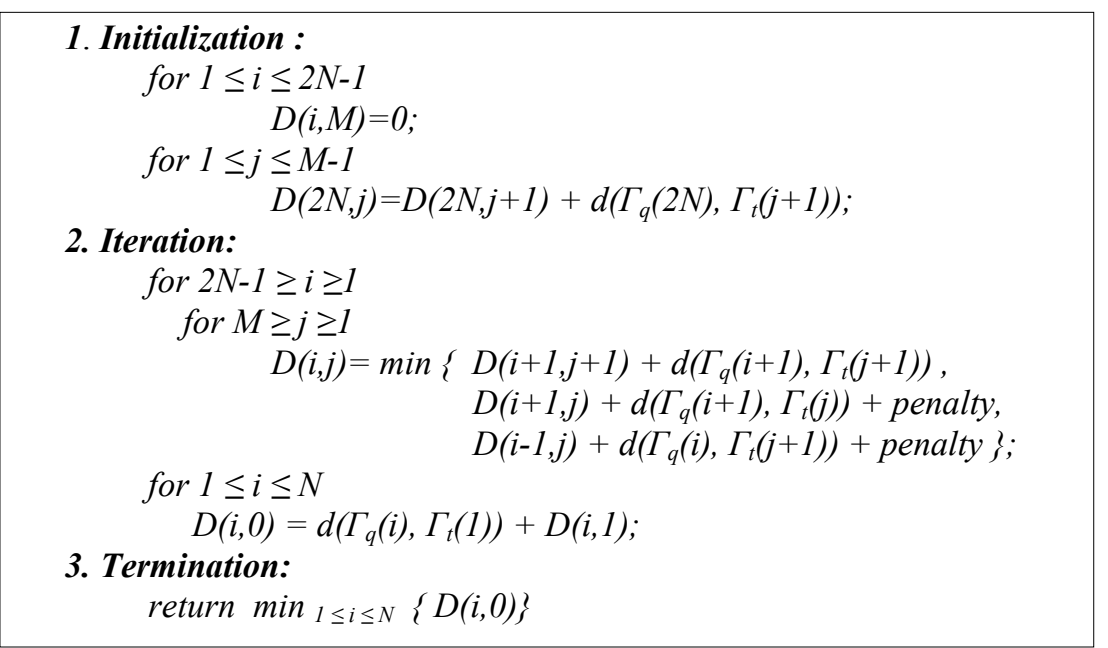

Note that, we change the recurrence relation by giving a constant penalty to the horizontal and vertical moves through the matrix. Although there is no theoretical reason for giving penalties, the discussion about this revision on the algorithm can be given by the following arguments:

Given two sequences $\mathrm{A}$ and $\mathrm{B}$, the algorithm given in [8], finds a subsequence $\mathrm{Z}$ of $\mathrm{A}^{2}$, which is most similar to $\mathrm{B}$. In the computation, there is no control over the length of minimum distance path. Therefore, the length of the subsequence $\mathrm{Z}, \mathrm{Z} \mid$ may tend to go far from $|\mathrm{A}|$. This leads to the following consequences in the matching process. The algorithm in [8] calculates a partial matching of A against B. However, our aim is to measure the overall distance between the sequences. This requires to find a complete correspondence between items of the sequences. The algorithm approximates the optimal solution and estimates a lower bound of the exact cyclic distance [7].

The proposed algorithm gives penalties for horizontal and vertical moves and controls the length of the path. This heuristic enforces the path to go through the diagonal and approximate $|\mathrm{Z}|$ to $|\mathrm{A}|$. By this way, the complete correspondence between the items of two sequences is computed and the optimal solution is approximated by stimulating the matching score. Another reason for using penalties lies within the same proposition as in the DW case.

\section{Experiments}

The performance of the BAS descriptor is tested in the data set of MPEG 7 Core Experiments Shape-1 Part B, which is the main part of the Core Experiments.

In the first set of experiments, the effect of modification in DW algorithm is tested. The exact cyclic distance between the BAS features is calculated by keeping one of 
the sequences fix and shifting the other one item at a time. The classical DW is recomputed over and over again. The minimum distance is taken as the exact distance between the sequences. The penalty value in DW recurrence relation is taken as 50 for this particular test data. The constant value used as a penalty in the algorithm improves the similarity rates almost $2 \%$ at all sampling rates. This is depicted in Table 1 .

Table 1. Comparison of DWP and DW Algorithms (\%).

\begin{tabular}{cccccc}
\hline \multirow{2}{*}{ Algorithm } & \multicolumn{5}{c}{ Length of Sequence } \\
\cline { 2 - 6 } & $\mathbf{1 0}$ & $\mathbf{2 0}$ & $\mathbf{3 0}$ & $\mathbf{4 0}$ & $\mathbf{5 0}$ \\
DW & 62.96 & 76.03 & 79.75 & 81.02 & 81.13 \\
DWP & $\mathbf{6 5 . 8 5}$ & $\mathbf{7 7 . 5 7}$ & $\mathbf{8 1 . 2 6}$ & $\mathbf{8 2 . 2 3}$ & $\mathbf{8 2 . 2 6}$ \\
\hline
\end{tabular}

Another set of experiments are performed in order to compare the proposed cyclic sequence comparison algorithm with the original one in [8]. The penalty value remains as 50 in this set of experiments. The proposed method outperforms the original algorithm almost $5 \%$ at all sampling rates.

Table 2. Comparison of Cyclic Sequence Comparison Algorithm and the algorithm proposed in $[8](\%)$.

\begin{tabular}{cccccc} 
Algorithm & \multicolumn{5}{c}{ Length of Sequence } \\
\cline { 2 - 6 } & $\mathbf{1 0}$ & $\mathbf{2 0}$ & $\mathbf{3 0}$ & $\mathbf{4 0}$ & $\mathbf{5 0}$ \\
Cyclic DTW & $\underline{\mathbf{6 3 . 3 5}}$ & $\frac{\mathbf{7 6 . 0 2}}{71.19}$ & $\frac{\mathbf{8 0 . 8 2}}{76.70}$ & $\frac{\mathbf{8 1 . 6 0}}{77.59}$ & $\frac{\mathbf{8 1 . 8 2}}{77.98}$ \\
\hline Partial Shape Matching & 59.15 & 71.19 &
\end{tabular}

In Table 3, the comparison of the BAS function with the recently reported results of [3] (Shape Context), [4] (Tangent Space), [5] (Curvature Scale Space), [9] (Zernika Moments), [10] (Wavelet) and [11] (Directed Acyclic Graph) is provided. As it is seen from the table, the proposed descriptor performs better then the bestperformance descriptors available in the literature, for the data set of MPEG CE Shape-1 part B.

Table 3. Comparison of Proposed Algorithms with BAS vector size 50 and recent studies .

\begin{tabular}{cccccccc}
\hline $\begin{array}{c}\text { Shape } \\
\text { Context }\end{array}$ & $\begin{array}{c}\text { Tangent } \\
\text { Space }\end{array}$ & CSS & $\begin{array}{c}\text { Zernika } \\
\text { Moment }\end{array}$ & Wavelet & DAG & $\begin{array}{c}\text { BAS } \\
\text { with } \\
\text { DWP }\end{array}$ & $\begin{array}{c}\text { BAS } \\
\text { with } \\
\text { CSS }\end{array}$ \\
\hline 76.51 & 76.45 & 75.44 & 70.22 & 67.76 & 60 & $\mathbf{8 2 . 2 6}$ & $\mathbf{8 1 . 8 2}$
\end{tabular}

\section{Conclusion}

In this study, we propose some improvements in two different similarity distance methods defined on the BAS feature space. The first method used for similarity measurement is Dynamic Warping (DW), which is widely used in speech recognition. The DW algorithm finds an optimal match between two sequences, which allows 
stretching and compression of sections in the subsequences. However, in shape boundary representation, the expansion and compression of some part of the sequence change the visual appearance of the shape. Therefore, the expansion and compression of BAS functions should be handled with a specific care, unlikely in speech case. For this reason, we propose to give penalties for subsequence expansion and compression of the BAS function. This simple modification of the cost function provides substantial improvement on the overall performance of the DW algorithm.

The second method is cyclic sequence matching algorithm, which approximates the optimal solution. To ensure a consistent description of a shape, which is cyclic in nature, a unique starting point must be defined for each shape. Since this is impractical to achieve, the alignment computation must determine the amount of cyclic shift that has taken place in order to find the optimal solution. However, this process increases the computational cost during the similarity measurement, resulting impractical computational cost for large databases. In order to avoid this complexity, we propose an efficient cyclic sequence comparison algorithm. In the performance evaluation of proposed methods, we use the dataset of MPEG 7 Core Experiments Shape- 1. It is observed that the proposed shape descriptor outperforms all the methods in the literature in this particular data set.

\section{References}

1. Arica N., Yarman-Vural F. T, BAS: A Perceptual Shape Descriptor Based On The Beam Angle Statistics, Pattern Recognition Letters, vol: 24/9-10, (2003) 1627-1639.

2. Sankoff D., Kruskal J., Time Warps, String Edits and Macromolecules, CLSI Publications, 1999.

3. Belongie, S., Malik, J., Puzicha, J., Shape Matching and Object Recognition Using Shape Contexts. IEEE Trans. PAMI, 24, 4, (2002) 509-522

4. Latecki, L. J., Lakamper, R., Shape Similarity Measure Based on Correspondence of Visual Parts. IEEE Trans. PAMI, 22, 10, (2000) 1185-1190.

5. Mokhtarian, F., Abbasi, S., Kittler, J., Efficient and Robust Retrieval By Shape Content Through Curvature Scale Space. Image Databases and Multimedia Search, A. W. M. smeulders and R. Jain ed., 51-58 World Scientific Publication (1997)

6. Bunke H., Buhler U., Applications Of Approximate String Matching To 2-D Shape Recognition, Pattern Recognition, 26 (12), 1797-1812, 1993.

7. Mollineda, R. A., Vidal E., Casacuberta F., Cyclic Sequence Alignments: Approximate Versus Optimal Techniques, International Journal Of Pattern Recognition and Artificial Intelligence, 16 (3), 291-299, 2002.

8. Gorman J. W., Mitchell O. R., Kuhl F, P., Partial Shape Recognition Using Dynamic Programming, IEEE Trans. PAMI, 10 (2), 257-266, 1988.

9. Khotanzad, A., Hong, Y. H., Invariant Image Recognition By Zernike Moments. IEEE Trans. PAMI, 12, (1990) 489-497.

10. Chuang, G., Kuo, C. - C., Wavelet Descriptor of Planar Curves: Theory and Applications. IEEE Trans. Image Processing, 5, (1996) 56-70.

11. Lin, L. -J., Kung, S. Y.,. Coding and Comparison of Dags as a Novel Neural Structure With Application To Online Handwritten Recognition. IEEE Trans. Signal Processing, (1996). 